\title{
Diagnosis and prediction of miscarriage: can we do better?
}

\author{
Florrie NY Yu ${ }^{1}$, MB, ChB, FHKAM (Obstetrics and Gynaecology), KY Leung ${ }^{1,2}$ *, MD, FRCOG \\ ${ }^{1}$ Department of Obstetrics and Gynaecology, Queen Elizabeth Hospital, Hong Kong \\ ${ }^{2}$ Department of Obstetrics and Gynaecology, Gleneagles Hong Kong-Hospital, Hong Kong \\ *Corresponding author: ky@kyleung.org
}

Hong Kong Med J 2020;26:90-2

https://doi.org/10.12809/hkmj205094

Miscarriage is the most common serious complication of pregnancy, occurring in approximately $20 \%$ of pregnancies. ${ }^{1}$ Miscarriage can cause anxiety and depression on the affected woman, and to the partner as well, albeit to a lower level. ${ }^{2}$

The ultrasound diagnosis of miscarriage has to be accurate. In 2011, a large multicentre study showed significant variation in the cut-off values for mean gestational sac diameter (MSD) and embryo crownrump length (CRL) used to define miscarriage. ${ }^{3}$ Some cut-off criteria were found to be potentially unsafe with a risk of inadvertent termination of a potentially viable pregnancy. ${ }^{3}$ Since then, cut-off values of MSD and CRL defining miscarriage have been changed in the United Kingdom and the United States to $\geq 25 \mathrm{~mm}$ (without an obvious yolk sac) and $\geq 7 \mathrm{~mm}$ (without fetal heart activity), respectively.,4 It was noted that in the guidelines for first-trimester ultrasound examination published by the Hong Kong College of Obstetricians and Gynaecologists in 2004, old cut-offs (20 mm for MSD and $5 \mathrm{~mm}$ for CRL) were used. ${ }^{5} \mathrm{~A}$ review of these cut-offs is required.

Transvaginal sonography is recommended to optimise the examination. Care must be taken when CRL measurement is close to any decision boundary for miscarriage or when MSD is being measured because of its high inter-observer limit of agreement, around $20 \% .{ }^{6}$ When a miscarriage is found by one examiner, a repeat scan by another examiner is a reasonable safeguard. ${ }^{4}$ A repeat scan $\geq 7$ days later will be appropriate if initial scan shows an embryo without heart activity or MSD $\geq 12 \mathrm{~mm}$ without embryo heart activity. ${ }^{4}$ A repeat scan $\geq 14$ days will be appropriate if MSD $<12 \mathrm{~mm} .{ }^{4}$

Among women with intrauterine pregnancy of uncertain viability (PUV), the miscarriage rate is $49.3 \%$ to $52 \%{ }^{7,8}$ Prediction of pregnancy outcome is a challenge and is necessary because it can assist counselling and decide frequency of follow-up ultrasonography. Demographic factors, ultrasound and biochemical markers either used alone or in combination have been described in the literature to predict miscarriage.

Advanced maternal age ( $\geq 35$ years) is a well-known risk factor because of the increase in chromosomal abnormalities with maternal age. Women who presented with vaginal bleeding, especially those having moderate or heavy bleeding, or blood clot per vagina were likely to subsequently miscarry. ${ }^{9}$ In this issue of the Hong Kong Medical Journal, Wan et $\mathrm{al}^{7}$ show similar findings. Interestingly, the authors found that moderate/ severe abdominal pain is a risk factor on univariate analysis, but this finding was not confirmed on multivariate analysis probably because vaginal bleeding was a cofounding factor. ${ }^{7}$

When ultrasound shows fetal cardiac activity, the subsequent rate of miscarriage is $5.2 \%$ to $10.4 \%{ }^{7,9,10}$ A meta-analysis of 18 eligible studies on ultrasound markers among 5584 women found that fetal bradycardia is the most significant marker, with a sensitivity of $84.2 \%$ in the prediction of miscarriage. ${ }^{11} \mathrm{~A}$ more recent study found that the combination of low fetal heart rate and small CRL increases the risk of subsequent pregnancy loss, from $5.0 \%$ to $21 \% .^{10}$ Because fetal heart rate varies with gestation, cut-offs for low fetal heart rate of $\leq 122$, $\leq 123$, and $\leq 158$ beats per minute for gestational weeks 6,7 , and 8 , respectively, have been proposed. ${ }^{10}$ Other investigators have suggested a single fetal heart rate cut-off at $\leq 110$ or 100 beats per minute to predict miscarriage. ${ }^{11,12}$

Other ultrasonographic markers associated with miscarriage include a small difference between MSD and CRL, ${ }^{13}$ and abnormal size of yolk sac. ${ }^{14}$ Using three-dimensional ultrasonography, small gestational sac volume (below the 5th percentile) is associated with risk of miscarriage with odds ratio of $5.25 . .^{15}$ In a recent study of 61 miscarriages, abnormal size of gestational sac and yolk sac appeared as early as 6 weeks of gestation, followed by abnormal changes in fetal heart rate and CRL at 7 and 8 weeks. ${ }^{14}$ Although subchorionic haematoma was found to be a predictor of miscarriage in a metaanalysis ${ }^{11}$ and in the study by Wan et $\mathrm{al}^{7}$ a recent study on pregnancies with detectable fetal heartbeat did not concur with these findings. ${ }^{10}$

A meta-analysis of 15 studies including 1263 women with threatened miscarriage found that serum CA 125 is the only serum marker that is useful in predicting outcome of a pregnancy with a viable fetus, whereas serum human chorionic gonadotropin and progesterone are not useful. ${ }^{16}$

Bottomley et $\mathrm{al}^{17}$ proposed a scoring system 
which included a combination of demographic and ultrasound variables to predict miscarriage. This scoring system can give an individualised probability of the pregnancy viability immediately following an ultrasound examination without the need of taking blood for biochemical markers and waiting for the results. In this study involving 1435 British women having detectable fetal heart activity and PUV, the use of this scoring system gave an area under the curve (AUC) of the receiver operating characteristic curve of $0.924 .{ }^{17}$ When this scoring system was validated, the accuracy was lower with AUC of 0.771 for the original study set of 376 women with PUV and AUC of 0.832 for another data set of 400 women with PUV. ${ }^{18}$ In their study, Wan et al report the first validation study of this scoring system on Chinese population, with AUC of 0.91 if only viable pregnancies were analysed. ${ }^{7}$ Although this scoring system is described as simple, ${ }^{7,17}$ its use requires extra time, and can be challenging to implement in a busy clinic setting. The use of this scoring system requires further studies in clinical settings.

Women with threatened miscarriage are at risk of anxiety and depression, ${ }^{19}$ and may react to miscarriage in different ways. ${ }^{20}$ Healthcare professionals should receive training on communication, and provide affected women with information and support in a sensitive and professional manner. ${ }^{18,20}$ During interpretation of ultrasound guidelines to diagnose miscarriage, other factors should be taken into consideration, including the woman's desire to continue their pregnancy or to postpone intervention to achieve total certainty of miscarriage, and their acceptance of disadvantages of such postponement including emergency admission or procedure for heavy vaginal bleeding and anxiety. ${ }^{12}$

In summary, it is important to avoid misdiagnosis of miscarriage by using updated protocols and repeating scans if in doubt. Appropriate counselling on pregnancy outcome can be given after assessment of maternal age, amount of vaginal bleeding, fetal heart rate, CRL, preference on continuing the pregnancy, and anxiety level.

\section{Author contributions}

All authors contributed to concept, analysis or interpretation of data, drafting of the manuscript, and critical revision of the manuscript for important intellectual content. All authors had contributed to the manuscript, approved the final version for publication, and take responsibility for its accuracy and integrity.

\section{Conflicts of interest}

All authors have disclosed no conflicts of interest.

\section{Funding/support}

This editorial received no specific grant from any funding agency in the public, commercial, or not-for-profit sectors.

\section{References}

1. Savitz DA, Hertz-Picciotto I, Poole C, Olshan AF. Epidemiologic measures of the course and outcome of pregnancy. Epidemiol Rev 2002;24:91-101.

2. Farren J, Mitchell-Jones N, Verbakel JY, Timmerman D, Jalmbrant M, Bourne T. The psychological impact of early pregnancy loss. Hum Reprod Update 2018;24:731-49.

3. Abdallah Y, Daemen A, Kirk E, et al. Limitations of current definitions of miscarriage using mean gestational sac diameter and crown-rump length measurements: a multicenter observational study. Ultrasound Obstet Gynecol 2011;38:497502.

4. Preisler J, Kopeika J, Ismail L, et al. Defining safe criteria to diagnose miscarriage: prospective observational multicentre study. BMJ 2015;351:h4579.

5. Hong Kong College of Obstetricians and Gynaecologists (HKCOG). Guidelines for first trimester ultrasound examination: Part I. HKCOG guidelines Number 10 Part I. Available from: http://www.hkcog.org.hk/hkcog/Download/ Guidelines_for_First_Trimester_Ultrasound_Exam_ Part1_2004.pdf. Accessed 9 Mar 2020.

6. Pexsters A, Luts J, Van Schoubroeck D, et al. Clinical implications of intra- and interobserver reproducibility of transvaginal sonographic measurement of gestational sac and crown-rump length at 6-9 weeks' gestation. Ultrasound Obstet Gynecol 2011;38:510-5.

7. Wan OY, Chan SS, Chung JP, Kwok JW, Lao TT, Sahota DS. External validation of a simple scoring system to predict pregnancy viability in women presenting to an early pregnancy assessment clinic. HKMJ 2020;26:102-10.

8. Bottomley C, Van Belle V, Pexsters A, et al. A model and scoring system to predict outcome of intrauterine pregnancies of uncertain viability. Ultrasound Obstet Gynecol 2011;37:58895.

9. Stamatopoulos N, Lu C, Casikar I, et al. Prediction of subsequent miscarriage risk in women who present with a viable pregnancy at the first early pregnancy scan. Aust N Z J Obstet Gynaecol 2015;55:464-72.

10. Devilbiss EA, Mumford SL, Sjaarda LA, et al. Prediction of pregnancy loss by early first trimester ultrasound characteristics. Am J Obstet Gynecol 2020 Feb 25. Epub ahead of print.

11. Pillai RN, Konje JC, Richardson M, Tincello DG, Potdar N. Prediction of miscarriage in women with viable intrauterine pregnancy-A systematic review and diagnostic accuracy meta-analysis. Eur J Obstet Gynecol Reprod Biol 2018;220:122-31.

12. Committee on Practice Bulletins-Gynecology. The American College of Obstetricians and Gynecologists Practice Bulletin no. 150. Early pregnancy loss. Obstet Gynecol 2015;125:125867.

13. Bromley B, Harlow BL, Laboda LA, Benacerraf BR. Small sac size in the first trimester: a predictor of poor fetal outcome. Radiology 1991;178:375-7.

14. Detti L, Francillon L, Christiansen ME, et al. Early pregnancy ultrasound measurements and prediction of first trimester pregnancy loss: a logistic model. Sci Rep 2020;10:1545.

15. Wie JH, Choe S, Kim SJ, Shin JC, Kwon JY, Park IY. Sonographic parameters for prediction of miscarriage: role of 3-dimensional volume measurement. J Ultrasound Med 2015;34:1777-84.

16. Pillai RN, Konje JC, Tincello DG, Potdar N. Role of serum biomarkers in the prediction of outcome in women with 
threatened miscarriage: a systematic review and diagnostic accuracy meta-analysis. Hum Reprod Update 2016;22:22839.

17. Bottomley C, Van Belle V, Kirk E, Van Huffel S, Timmerman $\mathrm{D}$, Bourne T. Accurate prediction of pregnancy viability by means of a simple scoring system. Hum Reprod 2013;28:6876.

18. Guha S, Van Belle V, Bottomley C, et al. External validation of models and simple scoring systems to predict miscarriage in intrauterine pregnancies of uncertain viability. Hum Reprod
2013;28:2905-11.

19. Zhu CS, Tan TC, Chen HY, Malhotra R, Allen JC, Østbye T. Threatened miscarriage and depressive and anxiety symptoms among women and partners in early pregnancy. J Affect Disord 2018;237:1-9.

20. National Institute for Health and Care Excellence (NICE). Ectopic pregnancy and miscarriage: diagnosis and initial management. NICE guideline [NG126]. Available from: https://www.nice.org.uk/guidance/ng126. Accessed $9 \mathrm{Mar}$ 2020. 